\title{
INTERPRETAÇÃO DA SENSIBILIDADE TUBERCULINICA EM POPULAÇÃO DO INTERIOR DO ESTADO DE SÃO PAULO*
}

Gilberto Ribeiro Arantes** Antonio Ruffino-Netto *** Jannete Nassar **

RSPU-B/315

\begin{abstract}
ARANTES, G.R, et al. - Interpretação da sensibilidade tuberculinica em população do interior do Estado de São Paulo. Rev. Saúde públ., S. Paulo, 10:219-26, 1976 .

RESUMo: O teste tuberculinico padronizado com $2 U . T$. de PPD rt 23 foi aplicado em 35.680 pessoas de todas as idades, ambos os sexos, residentes no municipio de Ribeirão Preto, São Paulo, região Sudeste do Brasil. O aspecto dos histogramas representativos da sensibilidade tuberculinica sugere a existência de infecçōes por micobactérias atipicas nessa área.
\end{abstract}

UNITERMos: Sensibilidade tuberculinica. Micobactérias atipicas. Teste tuberculinico.

\section{I N T RODUC $\mathrm{O}$}

Quando se analisa a distribuição dos diâmetros das reações tuberculínicas obtidas em uma população e dispostas na forma de histograma de freqüências relativas, é possivel obter diversas informações. Um primeiro enfoque permite aquilatar a qualidade técnica com a qual os testes foram lidos ${ }^{5}$. Todavia, mais importante é a possibilidade de se analisar o comportamento biológico da população face a infecções por micobactérias. Os padrões observados variam de região para região em decorrência da maior ou menor prevalência de reações fracas. Quando a prevalência destas é baixa, o aspecto do histograma é bimodal e bipartido ${ }^{4}$, tal como se vê na Figura $1 \mathrm{~A}$ adaptada de Edwards e Edwards ${ }^{3}$, o componente da esquerda tem u'a moda em torno de 0 a $2 \mathrm{~mm}$ caindo logo para a direita, e o componente da direita tem a forma de uma distribuição normal com u'a moda ao redor de 18 a $20 \mathrm{~mm}$; há um mínimo de superposição entre os dois componentes. Quando a prevalência de reações fracas é alta, o intervalo entre as duas modas é preenchido por um terceiro componente, esquematicamente apresentado na Figura $1 \mathrm{~B}$, e o histograma deixa de ser bipartido, podendo ocorrer uma série de padrões intermediários entre esses dois extremos. A experiência acumulada durante muitos

- Apresentado no XVII Congresso Nacional de Tuberculose e Doenças Respiratórias, Brasilia, D.F., 1975.

** Da 6.a Divisão Regional de Saúde da Secretarla da Saúde do Estado de São Paulo. Rua Minas, 895 - Rlbeirăo Preto, S.P. - Brasil.

** Da Faculdade de Medicina de Ribeirāo Preto da U.S.P. Fazenda Monte Alegre Ribeirão Preto, S.P. - Brasil. 
ARANTES, G. R. et al. - Interpretação da sensibilidade tuberculínica em população do interior do Estado de São Paulo. Rev. Saúde públ., S. Paulo, 10:219-26, 1976.

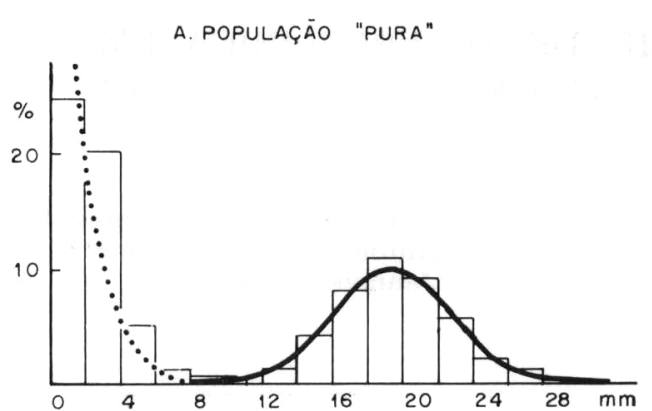

B. POPULAÇĀO "IMPURA"

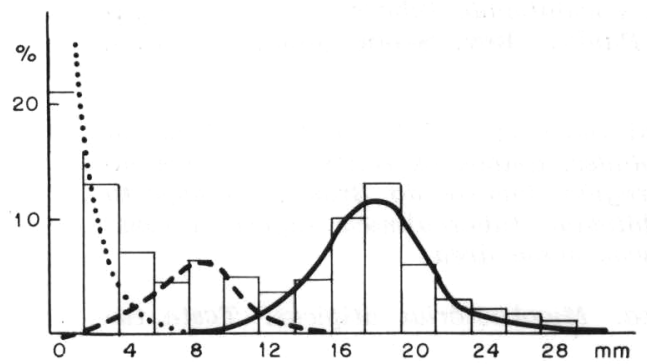

Fig. 1 ( $\mathrm{A}$ e B) - Distribuição dos diâmetros das provas tuberculínicas, PPD rt $5 \mathrm{U} . \mathrm{T}$. , com curvas esquemáticas mostrando os componentes em duas populacōes diferentes. Adaptada de Edwards \& Edwards 3

anos permite atribuir essas reações fracas à ocorrência de reaçōes cruzadas devidas a infecções por micobactérias atípicas. $\mathrm{Na}$ Figura 2, adaptada de Palmer e col. ${ }^{6}$, os histogramas correspondentes ao México e Dinamarca são expressivos de populações "puras", isto é, infectadas apenas pelo Mycobacterium tuberculosis, sendo a intensidade do problema tuberculose bem menor na Dinamarca. Em contrapartida os histogramas referentes ao Sudão e à Carolina do Norte são interpretados como expressão de populações "impuras", isto é, infectadas pelo $M$. tuberculosis e outras micobactérias; neste caso a prevalência de infecções inespecíficas seria maior no Sudão. De um modo geral o padrão correspondente a populaçōes "puras" tem sido encontrado nas regiões temperadas e sub- tropicais e o de populações "impuras" nas regiōes tropicais onde o "poder discriminador" do teste tuberculínico seria menor ${ }^{4}$.

O presente trabalho foi realizado com a finalidade de estudar a sensibilidade tuberculínica em regiấo onde o padrão esperado seria aquele correspondente a populações infectadas apenas pelo $M$. tuberculosis.

\subsection{Região estudada}

O município de Ribeirão Preto, Estado de São Paulo, região Sudeste do Brasil, situa-se a 518 metros de altitude, entre $21^{\circ} 10^{\prime} 42^{\prime \prime}$ de latitude sul e $47^{\circ} 48^{\prime} 24^{\prime \prime}$ de longitude W.Gr. Clima quente, inverno seco, oscilando as temperaturas entre mínima de $10^{\circ} \mathrm{C}$ e máxima de $34^{\circ} \mathrm{C}$ com média compensada de $21^{\circ} \mathrm{C}$. A precipitação pluvial anual é em média de 1.308 milímetros.

\section{MATERIAL E METODOS}

A população de estudo foi constituída pelas pessoas de qualquer idade, residentes no município de Ribeirão Preto, que se matricularam no Centro de Saúde Polivalente da cidade, para qualquer fim, durante 12 meses consecutivos, de maio de 1973 até abril de 1974.

$\mathrm{Na}$ Tabela 1 é apresentada a composição etária da população do município e da demanda estudada.

$\mathrm{Na}$ Tabela 2 é vista a distribuição da população geral e de demanda segundo o bairro de residência.

O teste tuberculínico foi aplicado segundo a técnica preconizada pela Divisão Nacional de Tuberculose ${ }^{2}$ em conformidade com a Organização Mundial da Saúde, por operadores padronizados segundo os critérios da Divisão Nacional de Tuberculose e supervisionados por en- 
ARANTES, G. R. et al. - Interpretação da sensibilidade tuberculinica em população do interior do Estado de São Paulo. Rev. Saúde públ., S. Paulo, 10:219-26, 1976.
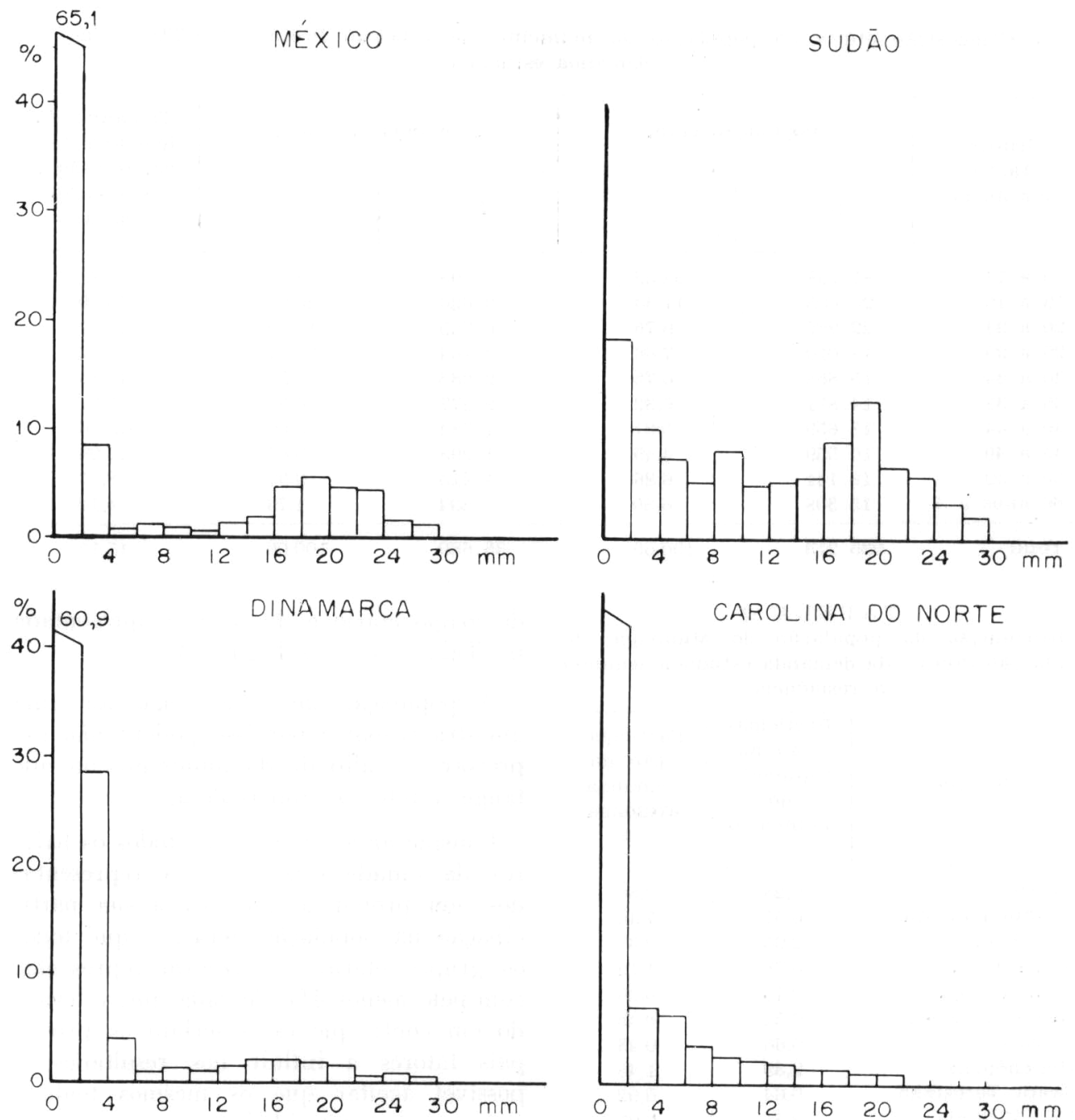

Fig. 2 - Distribuição dos diâmetros das provas tuberculínicas em escolares, PPD-S e PPD rt. Adaptada de Palmer e col. ${ }^{\circ}$.

fermeira de saúde pública aferida e padronizada naquela Divisão. A tuberculina usada foi o derivado protéico purificado conhecido como PPD, lote rt 23 , na dose de 2 unidades de tuberculina com 0,05 mil de Tween 80 , preparada no States Seruminstitut de Copenhague e diluída no Laboratório de Referência da Divisão Nacional de Tuberculose.

\section{RESLLTADOS E DISCUSSÃO}

De toda a população matriculada no Centro de Saúde no período assinalado, e portanto submetida à prova tuberculínica, voltaram para a leitura do teste 35.680 pessoas, isto é, $95 \%$ das matriculadas.

A distribuição dos diâmetros das endurações lidas nessa população, em função 
ARANTES, G. R. et al. - Interpretação da sensibilidade tuberculínica em populasão do interior do Estado de São Paulo. Rev. Saude públ., S. Paulo, 10:219-26, 1976.

TABELA 1

Composição etária da população do municipio de Ribeirão Preto (01/9/73) e da demanda estudada

\begin{tabular}{|c|c|c|c|c|c|}
\hline \multirow{2}{*}{$\begin{array}{c}\text { Grupos } \\
\text { etârios } \\
\text { (em anos) }\end{array}$} & \multicolumn{2}{|c|}{ População geral } & \multicolumn{2}{|c|}{ Demanda estudada } & \multirow{2}{*}{$\begin{array}{c}\text { Tamanho da } \\
\text { demanda (\%o) } \\
\text { em relação à } \\
\text { população } \\
\text { geral }\end{array}$} \\
\hline & N. ${ }^{\circ}$ & $\%$ & N.o & 9 & \\
\hline 0 a 14 & 81.208 & 34,52 & 4.798 & 13,45 & 5.91 \\
\hline 15 a 19 & 26.655 & 11,33 & 9.620 & 26,95 & 36,09 \\
\hline 20 a 24 & 22.952 & 9,76 & 6.705 & 18,79 & 29,21 \\
\hline 25 a 29 & 18.010 & 7,66 & 4.164 & 11,67 & 23,12 \\
\hline 30 a 34 & 15.868 & 6.75 & 2.985 & 8,37 & 18,81 \\
\hline 35 a 39 & 14.871 & 6,32 & 2.277 & 6.38 & 15,31 \\
\hline 40 a 44 & 13.659 & 5,81 & 1.784 & 5,00 & 13.06 \\
\hline 45 a 49 & 10.559 & 4,49 & 1.298 & 3,64 & 12,29 \\
\hline 50 a 59 & 16.141 & 6,86 & 1.415 & 3,97 & 8,77 \\
\hline 60 anos e + & 15.308 & 6,50 & 634 & 1,78 & 4,14 \\
\hline Total & 235.213 & 100,00 & 35.680 & 100,00 & 15,17 \\
\hline
\end{tabular}

TA B E L A 2

Distribuição da população do Município de Ribeirāo Preto e da demanda estudada segundo a residência

\begin{tabular}{|c|c|c|}
\hline Bairros & $\begin{array}{l}\text { Participa- } \\
\text { cão na } \\
\text { população } \\
\text { do } \\
\text { municipio } \\
\text { ø }\end{array}$ & $\begin{array}{c}\text { Participa- } \\
\text { cão na } \\
\text { demanda } \\
\text { estudada } \\
\%\end{array}$ \\
\hline Centro & 9,28 & 9,56 \\
\hline Jardim Paulista & 6,57 & 7,40 \\
\hline Vila Seixas & 2.95 & 2,38 \\
\hline Santa Cruz & 0,78 & 1,14 \\
\hline Jardim Irajá & 0,75 & 0,36 \\
\hline Jardim América & 0,54 & 0,45 \\
\hline Sumaré & 0,66 & 0,43 \\
\hline Higienópol،s & 2,39 & 1,46 \\
\hline Santa Terezinha & 0,64 & 0,92 \\
\hline Vila Vurginia & 9,55 & 9,95 \\
\hline Vila Monte Alegre & 0,93 & 0,79 \\
\hline Vila Lobato & 4,45 & 4,61 \\
\hline Vila Tibério & 8,93 & 9,25 \\
\hline Ipiranga & 8,54 & 10,62 \\
\hline Vila Recreio & 5,22 & 6,50 \\
\hline Campos Eliseos & 14.23 & 15,75 \\
\hline Vila Tamandaré & 3,39 & 3,54 \\
\hline Lapa & 5,64 & 6,21 \\
\hline Vila Carvalho & 3,33 & 4,57 \\
\hline Vila Abranches & 1,15 & 0,53 \\
\hline Vila Bonfim Pauista & 1,53 & 0,68 \\
\hline Vila Guatapará & 0,72 & 0,38 \\
\hline Zona Rural & 7,83 & 2,30 \\
\hline Prejudicado & - & 0,22 \\
\hline Total & 100,00 & 100,00 \\
\hline
\end{tabular}

do grupo etário e do sexo. é apresentada na Tabela 3 e na Figura 3.

A população de estudo não foi uma amostra casual e por isso poderá não expressar a realidade do município no que tange à infecção tuberculosa.

Considerando, porém, que todos os bairros da cidade estavam nela representados, em proporção idêntica a sua participação na população geral; e que todos os grupos etários se fizeram representar com pelo menos $4 \%$ de cada um; e levando em conta que esses seriam os principais fatores a influir nos resultados, é possivel aceitar que os mesmos tenham certa representatividade.

A análise dos histogramas da Figura 3 revela uma transição gradual e regular de uma coluna para outra, sem preferência por determinados valores, expressando um bom padrão técnico de leitura.

Os histogramas referentes aos grupos etários até 29 anos são semelhantes: elevada frequêencia de reaçôes de 0 - $4 \mathrm{mi}$ límetros que diminuem com o aumento da idade; queda brusca até a coluna correspondente a 6 e $7 \mathrm{~mm}$; a partir daí as freqüências rão diminuindo de forma 


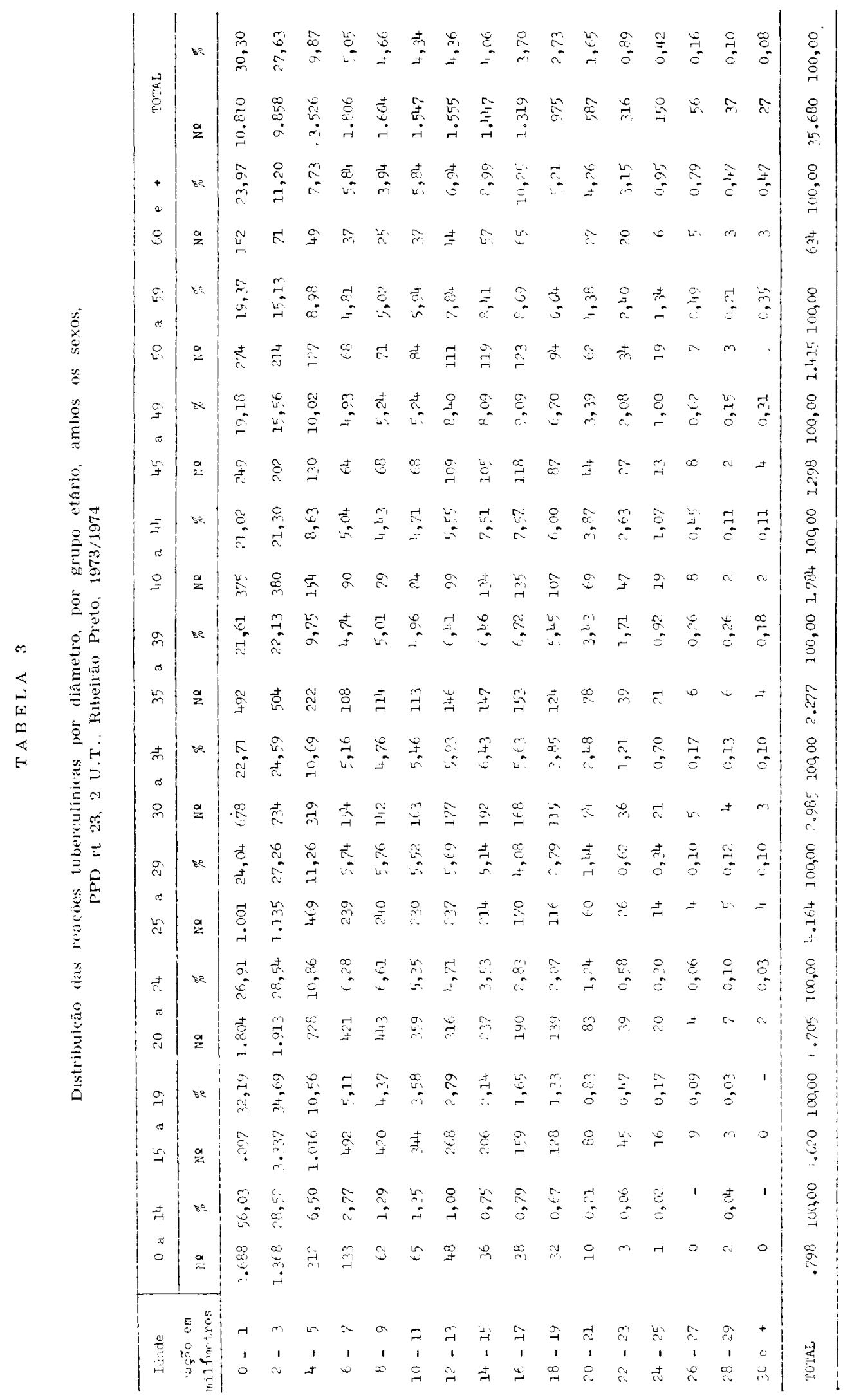


ARANTES, G. R. et al. - Interpretação da sensiblidade tuberculinica em população do interior do Estado de São Paulo. Rev. Saúde públ., S. Paulo, 10:219-26, 1976.

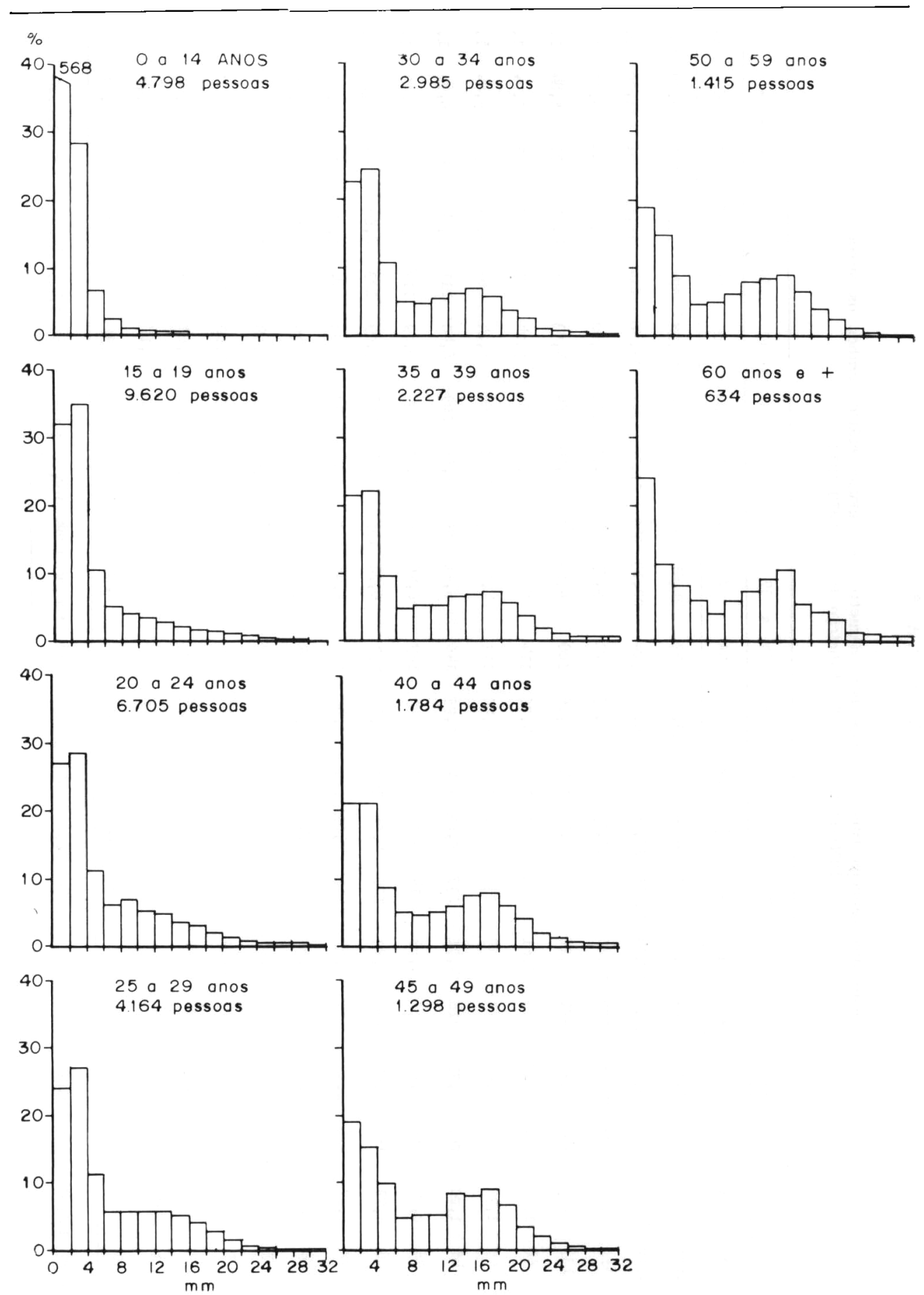

Fig. 3 - Distribuição dos diâmetros das provas tuberculínicas por grupo etário, ambos os sexos, PPD rt 23, 2 U.T., Ríbeirão Preto, 1973/74. 
ARANTES, G. R. et al. - Interpretação da sensibilidade tuberculínica em população do interior do Estado de São Paulo. Rev. Saúde públ., S. Paulo, 10:219-26, 1976.

suave e gradativa até a extremidade direita onde estão as freqüências correspondentes às maiores reações. Observa-se uma nítida diferença entre os não-reatores até $3 \mathrm{~mm}$ e os demais; a separação entre não-reatores e reatores está situada na coluna correspondente aos diâmetros 4. e $5 \mathrm{~mm}$. Em nenhum dos histogramas é possível distinguir o limite entre reatores fracos e fortes devido à existência de um número relativamente grande de reatores fracos dificultando essa distinção.

A partir do grupo etário 30 a 34 anos diminui a proporção de não-reatores elevando-se a de reatores fortes, variando pouco a de reatores fracos; esboça-se a partir desse grupo etário uma tendência à distribuição bimodal, que se acentua com a idade, embora sem o aparecimento de uma nítida área de separação, que lhe daria uma configuração bipartida ${ }^{4}$. Essa evolução dos histogramas sugere a ocorrência de destuberculização progressiva nessa população, os gráficos obtidos nas pessoas mais velhas representando situações epidemiológicas antigas, quando a prevalência da tuberculose era mais elevada.

Uma vez que o problema tuberculose existe em nosso meio, por que motivos os histogramas correspondentes aos grupos mais jovens não apresentam também uma configuração bimodal como acontece com o histograma da Figura 2 referente a escolares da Dinamarca?

O padrão observado nas crianças de Ribeirão Preto é muito parecido com o da Carolina do Norte (Figura 2) onde é alta a prevalência de micobactérias não classificadas. É possivel que o fato se deva à existência de infecções cruzadas por essas bactérias na área em pauta, as quais estariam preenchendo a zona intermediária do histograma e mascarando a verdadeira forma do componente da direita; esquematicamente seriam três os componentes desse histograma: $010^{\circ}$ à es- querda corresponderia a reações provocadas pela injeção do diluente; o $2 .^{\circ}$ intermediário, cuja forma seria a normal, devido a micobactérias anônimas; e o $3 .^{\circ}$, à direita, também com forma normal e devido a reações específicas. Como nesses grupos mais jovens, a terceira curva seria muito larga e baixa, a curva intermediária não deixaria entrever a sua forma verdadeira; nos grupos etários mais velhos, progressivamente mais numerosos

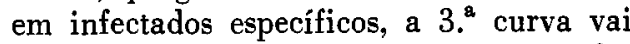
se tornando mais elevada (sem que tenha aumentado a proporção de reatores fracos) e sofrendo cada vez menos o efeito mascarador da curva intermediária de modo a permitir o aparecimento da bimodalidade, sem que no entanto ocorra uma nítida zona de clivagem. Obviamente entre os reatores fracos devem existir infectados específicos cuja proporção não pode ser estimada.

Se for correta essa interpretação, isto é, se existem infecções por micobactérias anônimas nessa área, o risco de contrair essas infecções não tem variado (a proporção de reatores fracos é praticamente a mesma em todos os grupos etários), sugerindo que a estrutura epidemiológica dessas infecções tem permanecido inalterada, parecendo-nos assim que no momento não constitui ou representa um problema de Saúde Pública.

Claro que a explicação aventada não é a única possível, embora seja a mais plausível à luz dos conhecimentos atuais.

Dado um histograma qualquer e supondo que o mesmo possa resultar de uma mistura de componentes correspondendo a diferentes características ligadas ao agente ou ao hospedeiro, é possivel separar matematicamente estes componentes. (Assim, por exemplo, Bhattacharya ${ }^{2}, 1967$ propõe um método para decomposição de uma mistura de distribuições).

Em distribuição de freqüências de eritema e de enduração obtidas por Ruffino- 
ARANTES, G. R. et al. - Interpretação da sensibilidade tuberculínica em população do interior do Estado de São Paulo. Rev. Saúde públ., S. Paulo, 10:219-26, 1976.

Netto ${ }^{7}$ (1970), Sanches ${ }^{8}$ (1963) aplicou o método de Bhattacharya mostrando que existiam 3 componentes para a enduração e 4 componentes para o eritema, assim como descreveu as características destas distribuições (média e desvios padrão).

Estas observações vêm de certa forma corroborar nossa hipótese anterior da existência de germes atípicos em nosso meio, apresentando um novo enfoque metodológico e quantificável.

$$
\text { 4. } \mathrm{CONCLUSAO}
$$

Do exposto anteriormente, somos propensos à aceitação da hipótese da existência de infecções por micobactérias anônimas na área de Ribeirão Preto.

ARANTES, G.R. et al. - [Tuberculin sensitivity in a community in the State of S. Paulo, Brazil]. Rev. Saúde públ., S. Paulo, 10:219-26, 1976

SUMMARY: The tuberculin test standardized with $2 T . U$. of PPD Rt 23 was applied to 35,680 people of all ages and both sexes, living in the municipality of Ribeirão Preto, São Paulo, Southeast region of Brazil. The aspect of the histograms representative of the tuberculin sensitivity suggests the existence of infections by Anonymous Mycobacteria in this area. test.

UNITERMS: Tuberculin sensitivity. Anonymous mycobacteria. Tuberculin

\section{REFERENCIAS BIBLIOGRAFICAS}

1. BHatTacharya, G.G. - A simple method of resolution of a distribuition into Gaussian components. Biometrics, 23:115-35, 1967.

2. CAMPANHA NACIONAL CONTRA A TUBERCULOSE. Comissão Técnica. Prova tuberculinica em saúde pública (2. recomendação). Rev. serv. nac. Tuberc., $12(47): 219-21,1968$.

3. EDWARDS, P.Q. \& EDWARDS, L.B. Story of the tuberculin test from an ep:demiologic view-point. Amer. Rev. resp. Dis., 81:1-47, 1960.

4. NYBOE, J, - The efficacy of the tuberculin test. Bull. Wld Hlth Org., 22: 5-37, 1960.

5. NYBOE, J. \& CHRISTENSEN, O.W. Measurement of the incidence of tuberculosis infection. Bull. Wld Hlth org., 35:547-55, 1966.
6. PALMER, C.E. et al. - Experimental and epidemiologic basis for the interpretation of tuberculin sensitivity. $J$. Pediat., St. Louis, 55:413-29, 1959.

7. RUFFINO-NETTO, A. - Epidemiologia da tuberculose: estudo de alguns aspectos mensuráveis na prova tuberculinica. Ribeirão Preto, 1970. [Tese de doutoramento - Faculdade de Medicina de Ribeirão Preto da USP].

8. SANCHES, O. - Extensão do método de BHATTACHARYA para decomposição de uma distribuiçāo de freqülências em componentes normais: aplicacão a um problema biológico. Ribeirão Preto, 1973. [Tese de doutoramento - Faculdade de Filosofia, Ciências e Letras de Ribeirão Preto].

Recebido para publicação em 13/01/1976 Aprovado para publicacāo em 12/03/1976 